\title{
Intramyometrial gestation: a rare localization of ectopic pregnancy
}

\begin{abstract}
Intramyometrial pregnancy is the rarest subtype of ectopic pregnancy. It poses a diagnostic and therapeutic challenge. Preoperative diagnosis is difficult and hysterectomy may be required due to delay in diagnosis. Misdiagnosis can lead to uterine rupture with life threatening haemorrhage. We report a rare case of intramyometrial ectopic in a 27year old lady, P3L3 with no history of previous abortions. Patient was admitted at 8 weeks of gestation with pelvic pain and haemorrhage. She underwent ultrasound pelvis and MRI pelvis which revealed an intramyometrial ectopic gestation. Findings on diagnostic laparoscopy revealed an asymmetrically enlarged anterior wall of uterus. Hysteroscopy was performed concurrently demonstrating an empty uterine cavity. Medical management was adopted with methotrexate. She was followed up until the Beta HCG levels returned to baseline. This case report illustrates the imaging findings of an exceptional case of intramyometrial ectopic pregnancy.
\end{abstract}

Volume 2 Issue 5 - 2017

\begin{abstract}
Ravikanth Reddy, Partha Sarathi Sarkar, Denver Steven Pinto, Babu Philip

St.John's Medical College, Rajiv Gandhi University of Health Sciences, India
\end{abstract}

Correspondence: Partha Sarathi Sarkar, Resident in
Radiology, St. John's Medical College, Bangalore 560034,Tel
+91886|522272, Email drparthasarathisarkar@gmail.com

Received: March 20, 2017| Published: April 05, 2017

Keywords: intramyometrial ectopic, ultrasound, MRI, methotrexate

\section{Introduction}

Following fertilization and fallopian tube transit, the blastocyst normally implants within the uterine cavity. Implantation at a site other than the uterine cavity is considered ectopic and comprises $1-2 \%$ of all first trimester pregnancies. ${ }^{1}$ Nearly $95 \%$ of ectopic pregnancies are implanted in various segments of the fallopian tube with the remaining $5 \%$ of non tubal pregnancies implanting in the ovary, peritoneal cavity, cervix or prior cesarean scar. Intramural pregnancy refers to a gestation completely implanted within the myometrium of the uterus separate from the uterine cavity, fallopian tube or round ligament. This is a very rare location for an ectopic and less than 50 cases have been reported in literature (less than $1 \%$ of all ectopic pregnancy). ${ }^{2}$ These cases are usually complicated by haemorrhage and uterine rupture. Diagnosis is often difficult with the closest differential being hydatidiform mole. Diagnostic modalities include ultrasound scan and magnetic resonance imaging demonstrating myometrium completely surrounding the gestational sac with no communication with the endometrial cavity. No documented case of term pregnancy does exist. However, if diagnosis is made earlier, treatment may be conservative, resulting in the preservation of reproductive potential. Treatment modalities documented in literature for intramural pregnancy include expectant management, surgical enucleation, uterine artery embolization, systemic or local methotrexate administration, hysterectomy and intrafetal injection of potassium chloride. ${ }^{3}$

\section{Case report}

We report a rare case of intramyometrial ectopic pregnancy in a 27year old lady, P3L3 who presented to the emergency department at 8 weeks of gestation with complaints of pelvic pain and haemorrhage not subsiding with medical management. Transvaginal ultrasound revealed an exocentric gestational sac with thick decidua reaction and a "Ring of fire" appearance on color Doppler. Uterine cavity was empty and no adnexal masses visualized. Furthermore, MRI of the pelvis with contrast was done to confirm the location of the ectopic gestation which revealed a well defined $\mathrm{T} 1$ isointense $\mathrm{T} 2$ hyperintense lesion in the anterior myometrium abutting the junctional zone and showing contrast enhancement. Endometrial stripe was seen separately with an empty uterine cavity. Pouch of Douglas had no free fluid. On diagnostic laparoscopy, an asymmetrically enlarged swollen anterior wall of the uterus was seen. Hysteroscopy was performed concurrently demonstrating an empty uterine cavity. Medical management with methotrexate was initiated following confirmation of the diagnosis. Beta HCG at the time of diagnosis was $9949 \mathrm{mIU} / \mathrm{ml}$. She was followed up until the hormone levels returned to baseline and serial scans showed a healed solid collection (Figures 1-7).
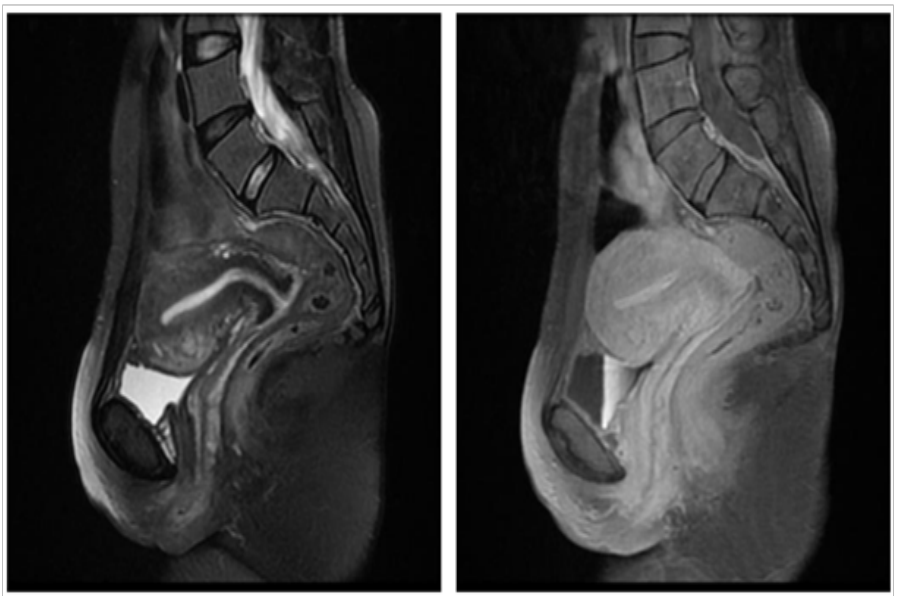

Figure I \& 2 Coronal T2W spin-echo image and TI+ Contrast image showing well defined hyperintense lesion involving the anterior myometrium showing homogenous contrast enhancement. 

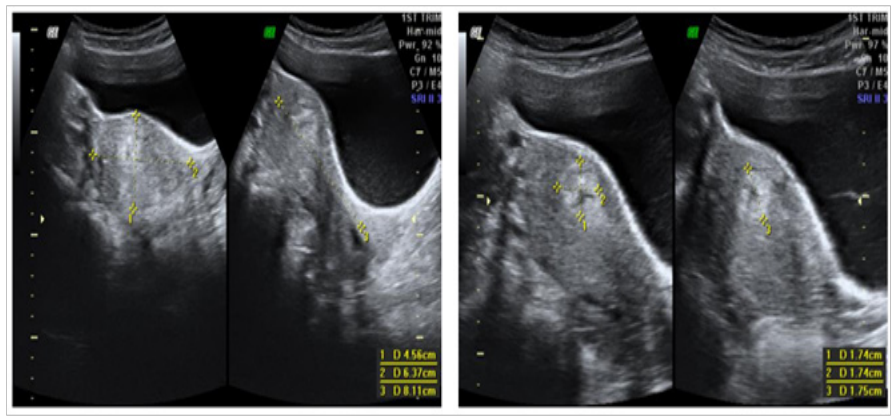

Figure 3 \& 4 Transabdominal ultrasound images showing well defined lesion in the anterior myometrium with thick decidual reaction. Endometrial stripe is separately visualized with empty uterine cavity.
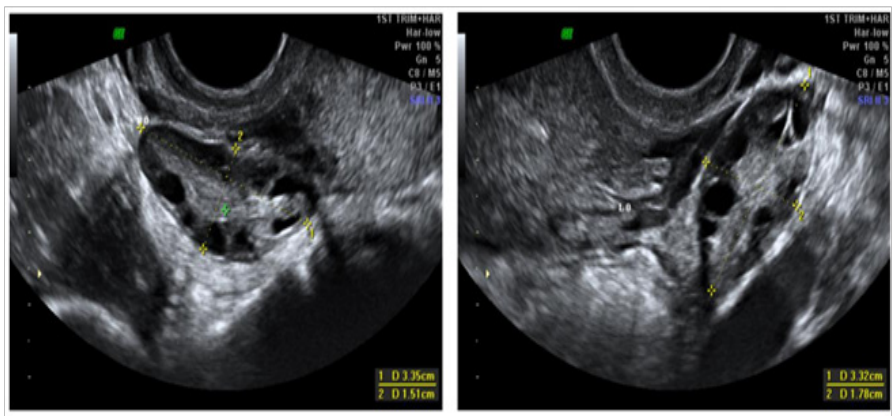

Figure 5 \& 6 Transvaginal ultrasound images showing Right and Left ovaries with no adnexal lesions.
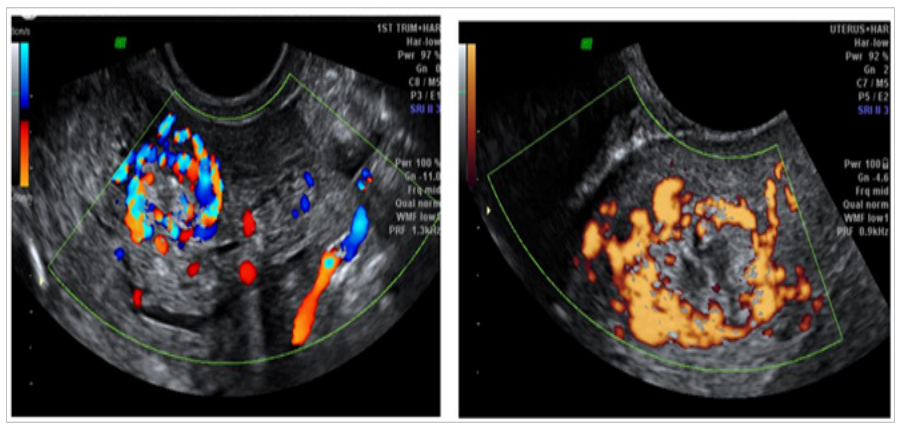

Figure 7 \& 8 Color doppler and power doppler transvaginal ultrasound images showing "Ring of fire" appearance suggesting hypervascularity of the intramyometrial ectopic.

\section{Discussion}

Ectopic pregnancy is the most common gynecologic abnormality and emergency that afflicts a potential mother and remains a major cause of mortality worldwide. It comprises $1-2 \%$ of all pregnancies with a myriad of locations having a hierarchy of prevalence as well as associated morbidity and mortality. ${ }^{4}$ commonest location for ectopic pregnancy continues to be the fallopian tube. Intramyometrial ectopic pregnancy constitutes less than $1 \%$ of their total number and is the rarest type. Pathological definition of an intramyometrial pregnancy refers to a conceptus implanting within a myometrium and separated from both the uterine cavity and tubes as well as surrounded by myometrium. Intramyometrial gestation rarely exceeds 12 weeks gestation with risk of uterine rupture and $2.5 \%$ of maternal mortality. ${ }^{5}$

Most common etiological factor is uterine trauma resulting in a sinus tract within the endometrium. Other factors include increased trophoblastic activity and defective decidualisation which allows the conceptus to penetrate into the myometrium. Implantation on the focus of adenomyosis may also account for this phenomenon as may serosal implantation of the conceptus following external migration. Other factors like in vitro fertilization, embryo transfer and traumatic factors such as dilatation an curettage (D\&C), cesarean section, myomectomy, manual removal of placenta have also been implicated in the etiology of intramyometrial gestation. ${ }^{6}$

Pelvic pain and uterine bleeding in the presence of a positive pregnancy test are the hallmarks of an ectopic pregnancy. Early diagnosis of intramometrial gestation is very difficult and mostly made intraoperatively. Only three cases of intramural pregnancy have been correctly diagnosed preoperatively by ultrasound and one by magnetic resonance imaging. ${ }^{7}$ A gestational sac completely surrounded by myometrium is the typical ultrasound appearance of an intramural pregnancy. Ultrasound typically shows a gestational sac with thick decidual reaction with "Ring of fire" appearance suggesting hypervascularity, empty uterine cavity and no adnexal lesions. MRI confirms the location of the gestational sac and demonstrates no communication with the endometrial cavity and provides better visualization of the myometrium and endometrium separately. Some authors reported the use of hysteroscopy which allows direct visualization of the uterine cavity and tubal ostium and confirms the absence of the conceptus in the uterine cavity. Serial Beta HCG assay has been reported to be useful for the diagnosis. ${ }^{8}$

Prognosis is very poor for fetuses in intramyometrial pregnancies. Treatment options depend on the time of diagnosis. In a hemodynamically stable patient who wants a future pregnancy, this condition can be managed conservatively by local administration of KCL or Methotrexate or by systemic methotrexate either in single dose or as multiple dose regimens. Non-radical surgical management by enucleation or wedge resection with myometrial reconstruction can be performed. Uterine rupture with hemodynamic instability warrants a hysterectomy.

\section{Conclusion}

Intramyometrial gestation is a very rare type of ectopic gestation and is a life threatening condition. Early detection of intramyometrial ectopic pregnancy is needed to prevent complications like uterine rupture and helps preserve fertility. Closest differential diagnosis is hydatidiform mole. Transvaginal ultrasound determines the localization of the gestational sac and choice of management.

\section{Ethical clearance}

Taken from Institutional Ethical Review Board, St. John's Medical College, Bangalore-560 034.

\section{Acknowledgements}

None.

\section{Conflict of interest}

Author declares that there is no conflict of interest.

\section{References}

1. Larraín D, Marengo F, Bourdel N, et al. Proximal ectopic pregnancy : a descriptive general population - based study and results of different management options in 86 cases. Fertil Steril. 2011;95(3):867-871.

2. Boukhanni L, Benkaddour YA, Bassir A, et al. A Rare Localization of Ectopic Pregnancy : Intramyometrial Pregnancy in Twin Pregnancy following IVF. Case Reports in Obstetrics and Gynecology. 2014;2014:1-2. 
3. Verghese T, Wahba K, Shah A. An interesting case of intramyometrial pregnancy. BMJ Case Rep. 2012; 2012:bcr1120115187.

4. Maria LPA, Larciprete G, Valli E, et al. Unusual Ectopic Pregnancy : Beyond the tubes. EC Gynecology. 2016;1(1):12-22.

5. Kirk E, Bottomley C, Bourne T. Diagnosing ectopic pregnancy and current concepts in the management of pregnancy of unknown location. Hum Reprod Update. 2014;20(2):250-261.

6. Çalifikan E, Lu Ç, Corakçi A. Expectant Management of an Intramural Ectopic Pregnancy in a Primigravid Woman. Journal of the Turkish-German Gynecological Association. 2008;9(4):234-236.
7. Leyder M, Vos M De, Dujardin M, et al. Intramyometrial ectopic pregnancy in an ICSI patient following uterine artery embolization. Reproductive Biomedicine Online. 2010;20(6):831-835.

8. Marotta M, Donnez J, Michaux N, et al. Spontaneous intramyometrial pregnancy mimicking an intramural myoma: a diagnostic challenge. $G y$ necological Surgery. 2016;9(4):439-444. 